POS PROCEEDINGS

\title{
Higgs physics possibilities at a muon collider
}

The manuscript has been modified on $1 / 4 / 2022$ to include a missing name on the authors list

\author{
L. Sestini, ${ }^{a, *}$ P. Andreetto, ${ }^{a}$ C. Curatolo, ${ }^{a, b}$ A. Gianelle, ${ }^{a}$ D. Lucchesi, ${ }^{a, b}$ \\ L. Buonincontri, ${ }^{a, b}$ M. Casarsa, ${ }^{c}$ N. Bartosik, ${ }^{d}$ N. Pastrone, ${ }^{d}$ F. Collamati, ${ }^{e}$ \\ M. Palmer, ${ }^{f}$ P. Sala,${ }^{g}$ C. Riccardi, ${ }^{h, i}$ I. Vai ${ }^{h, i}$ and C. Aimè ${ }^{h, i}$ \\ ${ }^{a}$ INFN Sezione di Padova, Padova, Italy \\ ${ }^{b}$ University of Padova, Padova, Italy \\ ${ }^{c}$ INFN Sezione di Trieste, Trieste, Italy \\ ${ }^{d}$ INFN Sezione di Torino, Torino, Italy \\ e INFN Sezione di Roma, Roma, Italy \\ ${ }^{f}$ BNL, Upton, New York, USA \\ ${ }^{g}$ INFN Sezione di Milano, Milano, Italy \\ ${ }^{h}$ INFN Sezione di Pavia, Italy \\ ${ }^{i}$ University of Pavia, Pavia, Italy \\ E-mail: lorenzo.sestini@pd.infn.it
}

Muon collisions at multi-TeV center-of-mass energies are ideal for studying the Higgs-boson properties. The large number of produced Higgs bosons will allow to measure its couplings to fermions and bosons with an unprecedented precision. At multi-TeV centre-of-mass energies, the double (triple) Higgs-boson production rate will be sufficiently high to directly measure the parameters of trilinear (quadrilinear) self-couplings, enabling the precise determination of the Higgs boson potential. In this contribution a study of the $\mu \mu \rightarrow H v v$ and $\mu \mu \rightarrow H H \nu v$ processes, where the Higgs bosons decay in two b-jets, is presented based on the full simulation of the detector with an evaluation of the beam-induced background.

40th International Conference on High Energy physics - ICHEP2020

July 28 - August 6, 2020

Prague, Czech Republic (virtual meeting)

\footnotetext{
${ }^{*}$ Speaker
} 


\section{Introduction}

The measurements of the Higgs boson couplings are fundamental to probe the Standard Model (SM) and to shed light on new physics scenarios. The Higgs self-couplings are of particular interest, since deviations of their measurements from the SM have deep implications on the Higgs potential shape and on the electroweak simmetry breaking mechanism [1]. Most of the Higgs couplings with fermions and vector bosons have already been measured at LHC, but future colliders could push their precision below the $1 \%$ scale [2]. Up to now there are no measurements of the Higgs potential, apart from the Higgs boson mass term. The trilinear $\left(\lambda_{3}\right)$ and quadrilinear $\left(\lambda_{4}\right)$ self-couplings cannot be measured at the LHC, due to the low cross-section of the $H H$ and $H H H$ productions. The CLIC collaboration demonstrated that a $e^{+} e^{-}$linear collider could measure $\lambda_{3}$ with a precision in the order of $10 \%$ using collisions with a centre-of-mass energy of $\sqrt{s}=1.5$ and $3 \mathrm{TeV}$ [3]. The quadrilinear coupling $\lambda_{4}$ is difficult to be measured even at the ambitious FCC-hh project, where a limit at $68 \%$ Confidence Level (CL) in the interval $[-2,+13]$ is expected to be set using $p p$ collisions at $100 \mathrm{TeV}$ [4].

In a multi-TeV muon collider a significant number of $\mu \mu \rightarrow H H \nu v$ and $\mu \mu \rightarrow H H H v \nu$ could be produced [5], allowing the measurements of $\lambda_{3}$ and $\lambda_{4}[6,7]$ with unprecedented precision. In this machine, clean events as in $e^{+} e^{-}$colliders are possible, but high collision energy as in hadron colliders could be reached, due to low radiation losses. Nevertheless several technological challenges for both machine and detectors should be faced in order to demonstrate its feasibility. In this paper the reconstruction of the Higgs boson decay to $b$-jets is discussed, and prospects on the measurements of the $H H$ cross-section and $\lambda_{3}$ are given.

\section{Beam-induced background and detector challenges}

The detector performance at a muon collider may be limited by the beam-induced background (BIB) [8]. The BIB is produced by the decays of muons in circulating beams, that generate electrons, positrons and neutrinos. Interactions of such particles with the machine and the machine-detector interface (MDI) can produce secondary particles like photons, neutrons or hadrons. Differently from other proposed future machines, the BIB at a muon collider shows unique features: particles can arrive to the interaction point (IP) from several meters of distance, and they are asynchronous with respect to the bunch-crossing [15]. The MDI, the detectors and the reconstruction algorithms should be developed in order to mitigate the BIB. As an example, the Muon Accelerator Program (MAP) collaboration [9] proposed to put two tungsten cone-shaped nozzle in order to reduce the BIB at the IP. Moreover, the detector could employ 5D sensors that can measure the energy, position and time in order to reject part of the BIB. The reconstruction algorithms should also be defined in order to reduce the combinatorial and fake objects produced by the $\mathrm{BIB}$, keeping the reconstruction performance at an adequate level. For these reasons and for the uniqueness of the muon collider environment, the physics performance should be assessed by studying the full simulation of the experiment. 


\section{Measurement of the $H \rightarrow b \bar{b}$ cross-section and Higgs coupling to $b$-quarks}

This study [10] has been performed with the full simulation of the $\mu \mu \rightarrow H(\rightarrow b \bar{b}) v v$ process at $\sqrt{s}=1.5 \mathrm{TeV}$, assuming the detector proposed by the MAP collaboration [9]. The signal and the irreducible inclusive background $\mu \mu \rightarrow q \bar{q} v v$ have been generated with Pythia 8 [11]. The $b$-jet reconstruction and identification have been studied for the first time at a muon collider, and they are still far from being fully optimized. The BIB is subtracted from the calorimeter on a statistical basis, and then the calorimeter clusters are put together into jets by using a cone algorithm with radius $R=0.5$. The jet reconstruction efficiency goes from $60 \%$ to $80 \%$ for jets with $p_{\mathrm{T}}>40$ $\mathrm{GeV}$, the loss of efficiency is explained by the removal, in the BIB subtraction procedure, of clusters belonging to the signal. The $b$-tagging is performed by reconstructing 3 -tracks secondary vertices inside the jet cone. The tagging efficiency is of about $60 \%$ and the misidentification, mainly due to combinatorial vertices produced by the BIB, is in the range 1-3\%. This performance have been obtained with the full simulation at $\sqrt{s}=1.5 \mathrm{TeV}$ and we assume the same performance as for $\sqrt{s}=3,10 \mathrm{TeV}$. This assumption is very conservative, because the BIB yield is expected to decrease at higher energies, since the muons lifetime in the laboratory system is longer. The expected relative uncertainty on the measured cross section is obtained assuming a counting experiment: $\frac{\Delta \sigma}{\sigma}=\frac{\sqrt{S+B}}{S}$ where $S$ and $B$ are the expected number of signal and background events, respectively. $S$ and $B$ are determined by considering the Pythia 8 cross-sections, the assumed integrated luminosity and the reconstruction efficiencies. The uncertainty on the Higgs coupling with $b$-quarks, $g_{H b b}$, has been obtained with the following formula:

$$
\frac{\Delta g_{H b b}}{g_{H b b}}=\frac{1}{2} \sqrt{\left(\frac{\Delta \sigma}{\sigma}\right)^{2}+\left(\frac{\Delta \frac{g_{H W W}^{2}}{\Gamma_{H}}}{\frac{g_{H W W}^{2}}{\Gamma_{H}}}\right)^{2}},
$$

where $\Delta \frac{g_{H W W}^{2}}{\Gamma_{H}}$, the uncertainty related to the Higgs coupling to the $W$ boson, is taken compatible with that found by CLIC [12]. If we assume to reconstruct the $W$ boson in the muon final state, this is motivated by the fact that the BIB is absorbed by the calorimeters and it is negligible in the muon detectors. The results of this study are reported in table 1 . The expected uncertainty is of the same order as that obtained by CLIC with $\sqrt{s}=1.5$ and $3 \mathrm{TeV}$ collisions.

\begin{tabular}{c|c|c|c|c|c|c|c}
\hline $\begin{array}{c}\sqrt{s} \\
{[\mathrm{TeV}]}\end{array}$ & $\begin{array}{c}\mathcal{L} \\
{\left[\mathrm{cm}^{-2} \mathrm{~s}^{-1}\right]}\end{array}$ & $\begin{array}{c}\mathcal{L}_{\text {int }} \\
{\left[\mathrm{ab}^{-1}\right]}\end{array}$ & $\begin{array}{c}\sigma \\
{[\mathrm{fb}]}\end{array}$ & $S$ & $B$ & $\frac{\Delta \sigma}{\sigma}$ \\
{$[\%]$} & $\begin{array}{c}\frac{\Delta g_{H b b}}{g_{H b b}} \\
{[\%]}\end{array}$ \\
\hline 1.5 & $1.25 \cdot 10^{34}$ & 0.5 & 203 & 5500 & 6700 & 2.0 & 1.9 \\
3.0 & $4.4 \cdot 10^{34}$ & 1.3 & 324 & 33000 & 7700 & 0.60 & 1.0 \\
10 & $2 \cdot 10^{35}$ & 8.0 & 549 & 270000 & 4400 & 0.20 & 0.91 \\
\hline
\end{tabular}

Table 1: Summary of the parameters used as inputs for the determination of the Higgs coupling to $b$ quarks. The assumed data taking time is $4 \cdot 10^{7} \mathrm{~s}$. Expected luminosities are taken from [9].

\section{Measurement of the $H H$ cross-section}

The measurement of the $H H$ cross-section is the first step for the determination of the Higgs trilinear coupling $\lambda_{3}$. In this preliminary study we consider the process $\mu \mu \rightarrow H(\rightarrow b \bar{b}) H(\rightarrow$ 
$b \bar{b}) v v$, with four $b$-jets in the final state, at $\sqrt{s}=3 \mathrm{TeV}$. The signal and the irreducible $\mu \mu \rightarrow b \bar{b} b \bar{b} v v$ background have been generated with WHIZARD [13]. Other background sources are considered negligible, since at least one $b$-tag per Higgs candidate is required. The full simulation has been performed with a detector similar to that proposed by CLIC [14], where the two tungsten nozzles have been inserted in the beam pipe, and the tracking detector geometry has been modified in analogy with the tracking system of the MAP detector. Jets are reconstructed with a particle flow algorithm, using both tracks and calorimeter clusters. As a preliminary consideration we assume that, thanks to the employment of the particle flow, the jet efficiency and resolution are unaffected by the BIB. However we assume that the BIB has an impact on the tagging efficiency, since it introduces noise in the secondary vertex finding. For this reason the BIB has not been included in the full simulation, but events are reweighted for the $b$-tagging efficiencies obtained in [10] in the presence of the BIB. At least four jets with $p_{\mathrm{T}}>20 \mathrm{GeV}$ are required, jet pairs are assigned to Higgs candidates in the way that the following figure of merit is minimized:

$$
F=\sqrt{\left(m_{12}-m_{H}\right)^{2}+\left(m_{34}-m_{H}\right)^{2}},
$$

where $m_{12}$ and $m_{34}$ are the dijet invariant masses and $m_{H}$ is the nominal Higgs boson mass. A display of a $H H$ event is shown in Fig. 1. Five different kinematic observables are given in input

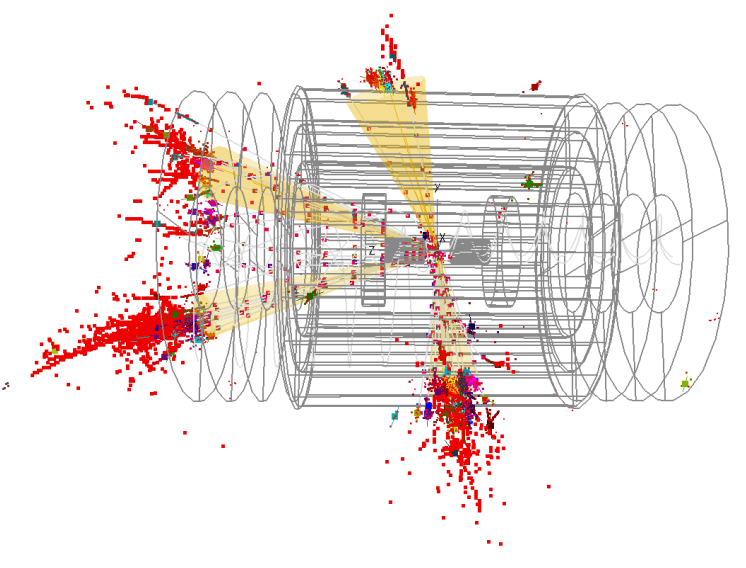

Figure 1: Display of a simulated $H(\rightarrow b \bar{b}) H(\rightarrow b \bar{b})$ process, BIB is not overlayed. The four $b$-jets are clearly visible.

to a multivariate discriminator, a Boosted Decision Tree, trained to separate the signal from the background. The five observables are: the reconstructed invariant mass of the highest $p_{\mathrm{T}}$ Higgs candidate and the one of the second Higgs candidate, the maximum angle between the jets, the total sum of the jets energy and the magnitude of the total jets momentum. The highest $p_{\mathrm{T}}$ Higgs mass and maximum angle distributions are shown in Fig. 2. The BDT distribution obtained with simulated data, and the expected yields for signal and background with an integrated luminosity of $1.3 \mathrm{ab}^{-1}$, are used to generate a pseudodataset for the BDT probability distribution. A number of $67 \mathrm{HH}$ events and 745 background events are expected. A fit to this distribution is performed to extract the uncertainty on the number of $\mathrm{HH}$ signal events, that is found to be $33 \%$. The output of the BDT and the fit result are shown in Fig. 3. This uncertainty is of the same order of that obtained by CLIC (20\%) in the same final state [12]. However this estimation is preliminary and will be improved in the future. 

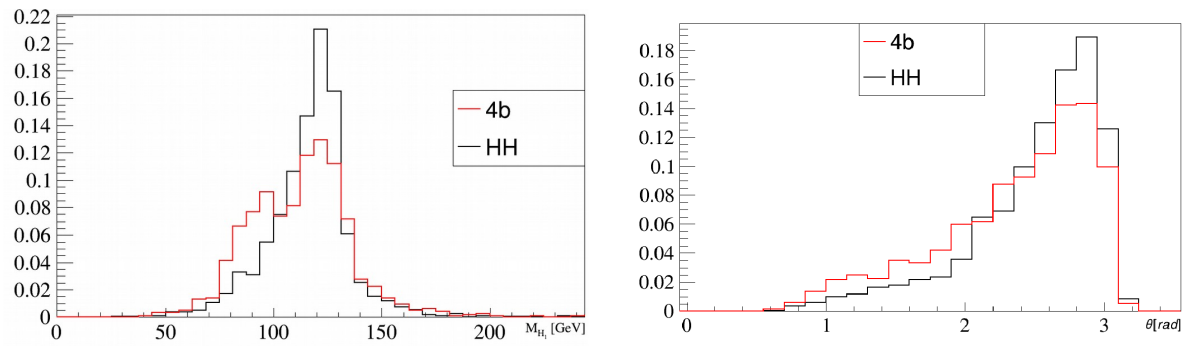

Figure 2: Distributions of (left) highest $p_{\mathrm{T}}$ Higgs mass and (right) maximum separation angle between jets for signal (red) and background (black).
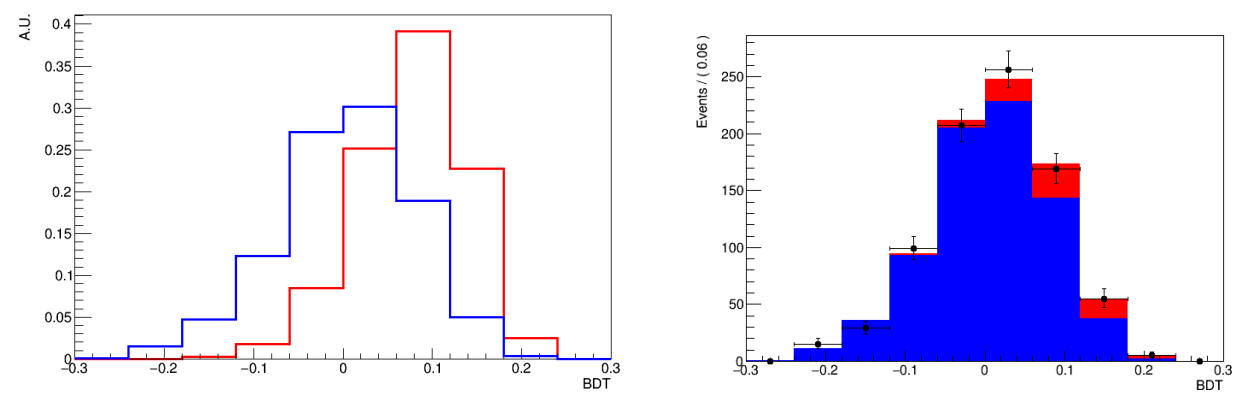

Figure 3: (Left) distribution of the BDT output and (right) fit result. The signal is coloured in red, the background in blue, black points with errors bands represent the pseudodataset.

\section{Prospects on the measurement of the trilinear coupling}

The dependence of the $H H$ cross-section at $\sqrt{s}=3 \mathrm{TeV}$ from the trilinear coupling obtained with WHIZARD is shown in Fig. 4, compared with the cross-section expected at the same $\sqrt{s}$ at CLIC [12]. It should be noticed that the $e^{+} e^{-}$cross-section is lower with respect to $\mu^{+} \mu^{-}$because of the different initial and final state radiation contributions. In order to measure the trilinear coupling, the expected number of $H H$ events at different $\lambda_{3}$ should be compared with the extracted number of events from the BDT fit. A $\chi^{2}$ technique could be employed to determine the confidence interval of the $\lambda_{3}$ measurement. In order to improve the sensitivity, the BDT could be trained to separate the background from the $H H$ component mediated by the trilinear coupling, instead of using the complete $H H$ sample. In conclusion, the muon collider is the ideal machine for Higgs physics, but technological challenges on the detector should be addressed. In this paper the feasibility of the measurements that involve the $H \rightarrow b \bar{b}$ decay has been demonstrated. New studies are in progress to determine the sensitivity on the Higgs self-couplings.

\section{References}

[1] P.A. Zyla et al., The Review of Particle Physics, Prog. Theor. Exp. Phys. 2020, 083 C01 (2020)

[2] J. de Blas et al., Higgs Boson studies at future particle colliders, JHEP 01 (2020) 139 


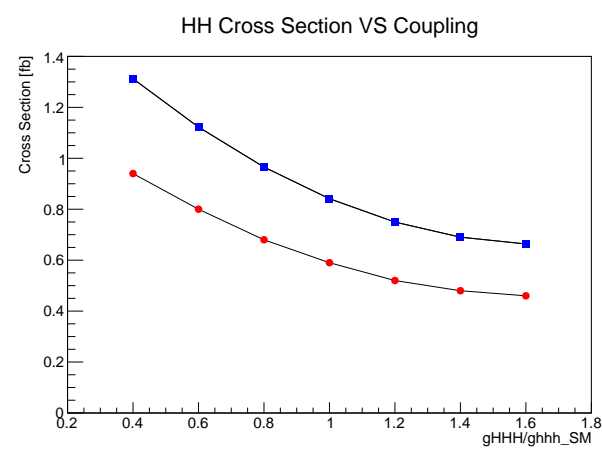

Figure 4: $H H$ cross section as a function of the trilinear coupling in muon-muon collisions obtained with WHIZARD (blue points) compared with the expectation obtained by CLIC in electron-positron collisions (red points). In the $x$ axis the value of the trilinear coupling is normalized to the Standard Model one.

[3] P. Roloff et al., Double Higgs boson production and Higgs self-coupling extraction at CLIC, Eur.Phys.J.C 80 (2020) 11, 1010

[4] A. Papaefstathiou et al., Triple Higgs boson production to six b-jets at a $100 \mathrm{TeV}$ proton collider, Eur. Phys. J. C (2019) 79: 947

[5] CLIC collaboration, The Compact Linear Collider (CLIC) - 2018 Summary Report, CERN2018-005-M

[6] A. Conway et al., Measuring the Higgs Self-Coupling Constant at a Multi-TeV Muon Collider, arXiv: 1405.5910

[7] M. Chiesa et al., Measuring the quartic Higgs self-coupling at a multi-TeV muon collider, JHEP 09 (2020) 098

[8] N. Bartosik et al., Preliminary Report on the Study of Beam-Induced Background Effects at a Muon Collider, arXiv:1905.03725

[9] Muon Accelerator Program, https://map.fnal.gov

[10] N. Bartosik et al., Detector and Physics Performance at a Muon Collider, 2020 JINST 15 $\mathrm{P} 05001$

[11] T. Sjöstrand et al., An Introduction to PYTHIA 8.2, 10.1016/j.cpc.2015.01.024

[12] H. Abramowicz et al., Higgs Physics at the CLIC Electron-Positron Linear Collider, Eur. Phys. J. C 77, 475 (2017)

[13] J. Reuter et al., Modern Particle Physics Event Generation with WHIZARD, arXiv:1410.4505

[14] L. Linssen et al., Physics and Detectors at CLIC: CLIC Conceptual Design Report, arXiv: 1202.5940

[15] M. Casarsa et al., Detector Performance Studies at a Muon Collider, Proceedings of 40th International Conference on High Energy physics (ICHEP2020), PoS(ICHEP2020)826 083. 